\title{
Optical Mass Gauging System for Measuring Liquid Levels in a Reduced Gravity Environment
}

\author{
Ryan M. Sullenberger, Wesley M. Munoz’ Matt P. Lyon, Kenny Vogel; and Azer P. Yalin ${ }^{\S}$ \\ Colorado State University, Fort Collins, CO, 80523
}

Valentin Korman

K Sciences, Huntville, Alabama 35814

Kurt A. Polzin"

NASA-Marshall Space Flight Center, Huntsville, AL 35812

\begin{abstract}
A compact and rugged fiber-coupled liquid volume sensor designed for flight on a sounding rocket platform is presented. The sensor consists of a Mach-Zehnder interferometer capable of measuring the amount of liquid contained in a tank under any gravitational conditions, including a microgravity environment, by detecting small changes in the index of refraction of the gas contained within a sensing region. By monitoring changes in the interference fringe pattern as the system undergoes a small compression provided by a piston, the ullage volume of a tank can be directly measured allowing for a determination of the liquid volume. To demonstrate the technique, data are acquired using two tanks containing different volumes of liquid, which are representative of the levels of liquid in a tank at different time periods during a mission. The two tanks are independently exposed to the measurement apparatus, allowing for a determination of the liquid level in each. In a controlled, laboratory test of the unit, the system demonstrated a capability of measuring a liquid level in an individual tank of $10.53 \mathrm{~mL}$ with a $2 \%$ error. The overall random uncertainty for the flight system is higher than that one test, at $\pm 1.5 \mathrm{~mL}$.
\end{abstract}

\section{Nomenclature}

molar refractivity, $\mathrm{m}^{3} / \mathrm{mol}$

length of the sensing path, $m$

optical refractive index

pressure, $\mathrm{Pa}$

universal gas constant, $\mathrm{J} /(\mathrm{K} \cdot \mathrm{mol})$

temperature of working gas, $\mathrm{K}$

$V_{\text {Cell }}$ volume of the reference gas cell, $\mathrm{mL}$
$V_{\text {Tank }}$

$V_{P}$

$\Delta m$

$\Delta n$

$\Delta p_{\text {Ref }}$

$\Delta p_{\text {Tank }}$

$\Delta \Phi$ tank ullage, $\mathrm{mL}$

volume of piston, $\mathrm{mL}$

fringe shift

change in refractive index

reference pressure change, $\mathrm{Pa}$

tank ullage pressure change, $\mathrm{Pa}$

change in phase, rad

\section{Introduction}

$\mathrm{T}^{\mathrm{H}}$

HE lack of gravity in the space environment makes it challenging to measure the amount of liquid propellant remaining in storage tanks. As shown in Fig. 1, liquid under microgravity conditions is free to float within the

${ }^{*}$ Undergraduate Student, Mechanical Engineering, Student member AIAA.

${ }^{\dagger}$ Undergraduate Student, Mechanical Engineering.

¥Undergraduate Student, Mechanical Engineering.

$\S$ Associate Professor, Mechanical Engineering.

T President and Director of Research.

॥ Propulsion Research Engineer, Propulsion Research and Technology Applications Branch, Propulsion Systems Department. Senior Member AIAA. 
confines of its containment system. Various mass gauging systems have previously been attempted. ${ }^{1-5}$ Volume compression/expansion systems either require complicated methods to avoid the acoustic sensitivities of a pressure transducer or require large displacement volumes. ${ }^{1}$ Other methods avoid the complications of reduced gravity effects by applying ground-based level measurements to a spacecraft, ${ }^{2}$ but this requires the craft to be under acceleration to 'settle' the fluid. Currently there are no options that have the capability to determine the liquid volume in a microgravity environment without adding significant mass or complexity to the propellant tank or imposing requirements on mission flight parameters.

An optical mass gauging system equipped with a modified Michelson interferometer was recently developed at NASA's Marshall Space Flight Center (MSFC) and has been shown as a promising option to measure liquid volumes with high precision using a small, lightweight system. ${ }^{6}$ The optical mass gauge is capable of monitoring critical onorbit storables, such as cryogenic fluids (fuels or oxidizers), water, and other liquids. The present paper describes recent efforts to modify the aforementioned optical mass gauging system to be compact and lightweight by utilizing a Mach-Zehnder interferometer that is fiber-coupled to a light source and detector. The use of fiber-optics in place of traditional optics (mirrors and beam splitters) allows the sensor to be ruggedized to withstand the extreme vibrations and accelerations experienced during launch and flight. The new sensor was fabricated as a payload to be flight tested on a sounding rocket platform. The apparatus has been extensively tested in the lab to ensure successful operation under flight conditions.

The outline for the rest of this paper is as follows. The technique of interferometric measurement of gas properties (specifically pressure) is presented in Sect. II. The design of the fiber-coupled optical mass gauge sensor is given in Sect. III. Testing results are presented and discussed in Sect. IV. Finally, suggested future improvements to the optical mass gauging system are outlined in Sect. V.
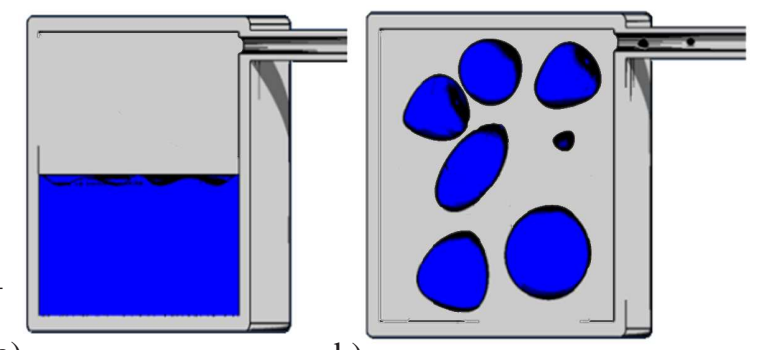

a)

b)

Figure 1. A tank containing a liquid a) under Earth gravity conditions and b) in a microgravity environment.

\section{Interferometric Measurement of Gas Properties}

The amount of liquid contained within a tank can be determined using an interferometer to measure the change in the index of refraction of the gas occupying the tank's ullage volume. A change in refractive index occurs as the gas is either compressed or expanded through the motion of a piston, changing the gas density (and also the pressure) and causing a shift in the interference pattern produced by the interferometer. In a Mach-Zehnder interferometer, a beam of light is first split into two equal halves. One path represents a reference beam that continues unaltered while the other beam passes through the medium being measured (sensing region). As the latter beam passes through the medium, a phase difference arises between the two beams. The phase difference between the two beams is a function of the gas density, as this affects the index of refraction.

Recombination of the two beams produces an interference pattern, which can be subsequently observed by pointing a photo-detector at one point on the pattern. The photo-detector measures the time-varying phase change between the two beams based on the passage of interference fringes (sequences of bright and dark regions) in front of the detector, which changes as the density in the gas varies. For a Mach-Zehnder interferometer, a $\Delta n$ change in the index of refraction yields a $\Delta m$ change in the interference order given by the equation: ${ }^{7}$

$$
\Delta m=\frac{\Delta \phi}{2 \pi}=\frac{l \Delta n}{\lambda},
$$

where $l$ is the length of the sensing region, $\Delta n$ is the change in refractive index of the gas in the sensing region, and $\lambda$ is the wavelength of light being used. Measuring the ullage of a tank requires the ability to measure a pressure change 
by examining the fringe shift. Using the Lorentz-Lorenz relation and assuming an ideal gas equation of state, the rate of change of pressure with respect to the order of interference is given as: ${ }^{7}$

$$
\frac{\partial p}{\partial m}=\frac{2 R T \lambda}{3 A l}
$$

where $R$ is the universal gas constant, and $T$ and $A$ are the temperature and molar refractivity, respectively, of the gas. Similar methods have demonstrated high volume sensitivity in other applications. ${ }^{8}$

\section{Mass Gauging Experiment Description}

\section{A. Sensor Design}

A fiber-coupled Mach-Zehnder interferometer is used to perform measurements on a gas cell, which is connected to a piston and two liquid tanks. The system operates on a single tank at a time. For demonstration purposes we employed two liquid tanks containing different volumes of water (representing tank levels at different periods during a mission) that can be independently exposed to the measurement apparatus such that the liquid volume in each can be determined. A generalized schematic of the optical mass gauge system is shown in Fig. 2.

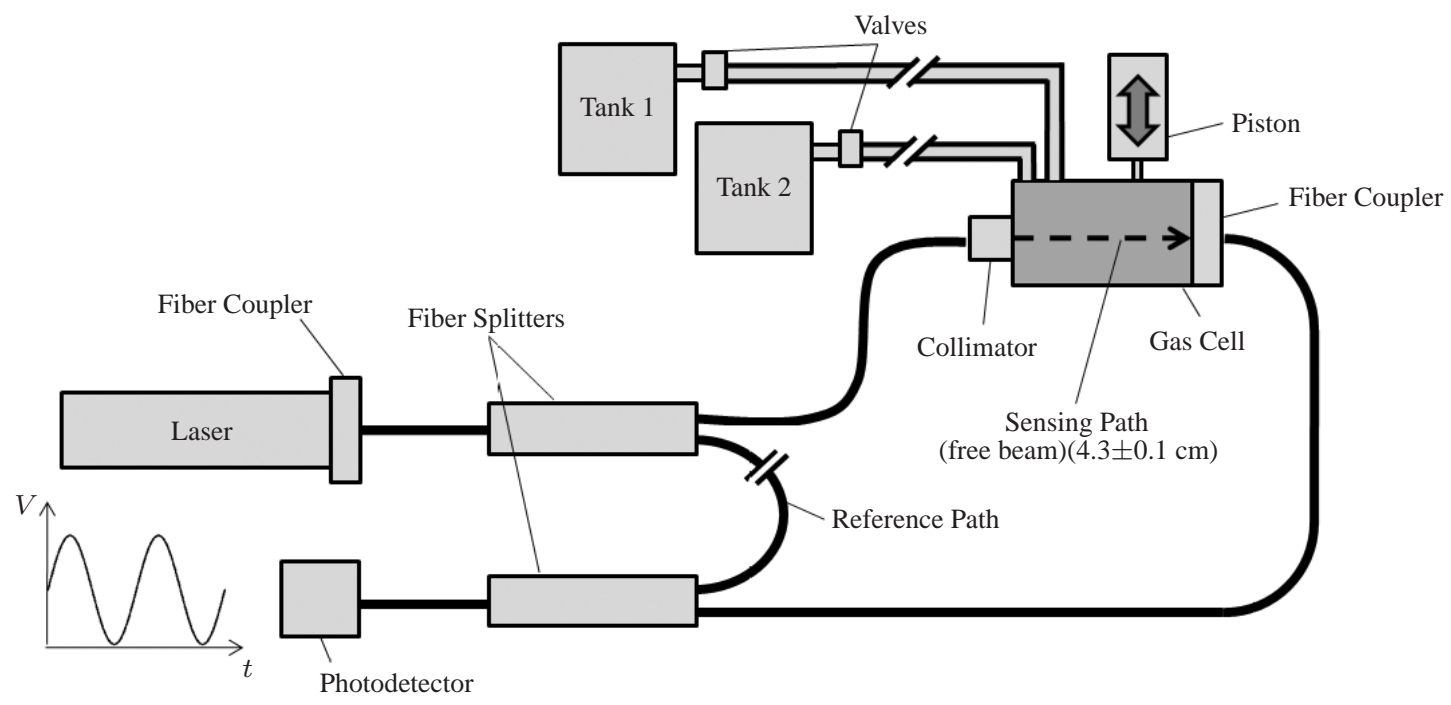

Figure 2. Schematic of a fiber-coupled optical mass gauging system.

Initially, both tanks are isolated from the gas cell and the system undergoes a cycling to provide a reference measurement. During the reference cycle, when the piston compresses its volume ("closed" position), the measured gas occupies the volume $V_{C e l l}$, which is comprised of the cell and associated gas lines. When the piston is moved allowing the gas to expand ("open" position), the same mass of gas additionally occupies the volume $V_{P}$ swept by the piston stroke (i.e. a total volume of $V_{P}+V_{C e l l}$ ). The actuation of the piston causes a shift in the interference fringes at the detector. The number of fringes that pass as the volume transitions from $V_{C e l l}$ to $V_{P}+V_{C e l l}$ is designated as the reference fringe count $\Delta m_{R e f}$. The value of $\Delta m_{R e f}$ can be related to the corresponding pressure change of the gas through Eq. 2. In the reference state, the pressure levels in the open and closed configurations $\left(p_{R e f, O p e n}\right.$ and $p_{\text {Ref,Closed }}$ respectively) are given as:

$$
\begin{gathered}
p_{\text {Ref }, \text { Open }}=\frac{n_{1} R T}{V_{\text {Cell }}+V_{P}}, \\
p_{\text {Ref,Closed }}=\frac{n_{1} R T}{V_{\text {Cell }}},
\end{gathered}
$$

where $n_{1}$ is the number of moles residing in the closed system. Assuming isothermal conditions, the system experiences the following pressure change during the reference cycle: 


$$
\Delta p_{\text {Ref }}=p_{\text {Ref }, \text { Closed }} \cdot\left(\frac{V_{P}}{V_{\text {Cell }}+V_{P}}\right) .
$$

For tank volume measurements, the targeted tank (with unknown ullage volume $V_{\text {Tank }}$ ) is exposed to the gas cell and piston volume and a second piston cycle is performed, producing a second fringe shift $\Delta m_{\text {Tank }}$. Fringe shift $\Delta m_{\text {Tank }}$ corresponds to a pressure change in the system in the same manner described above, yielding:

$$
\Delta p_{\text {Tank }}=p_{\text {Tank }, \text { Closed }} \cdot\left(\frac{V_{P}}{V_{\text {Tank }}+V_{\text {Cell }}+V_{P}}\right)
$$

where

$$
p_{\text {Tank }, \text { Closed }}=\frac{n_{2} R T}{V_{\text {Tank }}+V_{\text {Cell }}},
$$

and $n_{2}$ is the number of moles of gas in the full volume. Before any piston cycle is performed, gas pressure within all compartments of the system is equalized by opening and closing all solenoid valves so that:

$$
p_{\text {Ref,Closed }}=p_{\text {Tank,Closed }} .
$$

The $p_{\text {Tank,Closed }}$ and $p_{R e f, C l o s e d}$ terms are eliminated when taking a ratio of the two pressure changes. The pressure changes can be related to the fringe shifts (by Eq. 2) and:

$$
\frac{\Delta p_{\text {Ref }}}{\Delta p_{\text {Tank }}}=\frac{\Delta m_{\text {Ref }}}{\Delta m_{\text {Tank }}}=\frac{V_{\text {Cell }}+V_{\text {Tank }}+V_{P}}{V_{\text {Cell }}+V_{P}} .
$$

The ullage of the tank may be calculated knowing the volumes of the gas cell and piston, the reference fringe shift, and the new fringe shift. Knowledge of the theoretical volume of the entire tank allows for calculation of the volume of the liquid. Volumes of the gas cell and tanks are initially estimated and later determined experimentally.

\section{B. Hardware and Layout}

Drawings and a photograph of the optical mass gauge payload can be found in Fig. 3. The optical mass gauge sensor was designed as a sounding rocket payload and was subject to the constraints that the entire apparatus could be no larger than $23.62 \mathrm{~cm}$ in diameter and $12.1 \mathrm{~cm}$ tall, and mass limit was $2.97 \mathrm{~kg}$. The final sensor design fits within the required dimensions and massed $2.90 \mathrm{~kg}$.
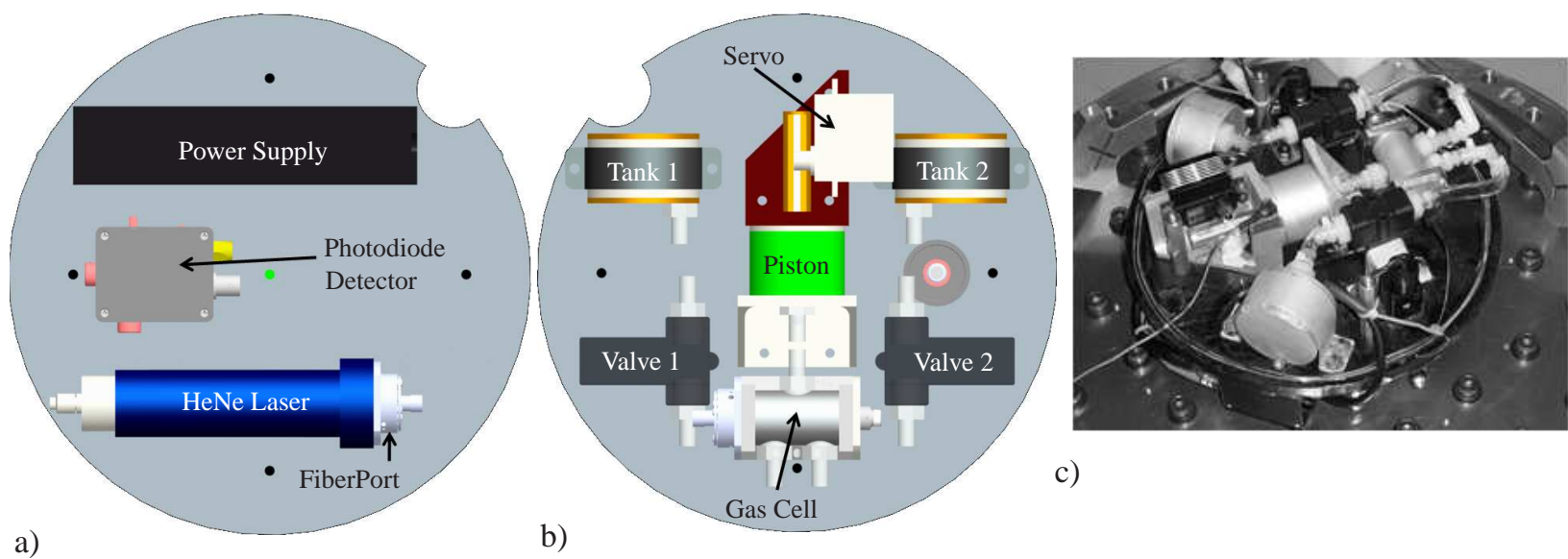

c)

Figure 3. Solid model drawings of a) the bottom and b) the top of the sensor, and c) the fully-assembled unit.

To withstand the extreme accelerations and vibrations of a typical launch, the sensor and light source were coupled using fiber-optic components. The use of fiber optics greatly increases the likelihood of maintaining optical alignment after launch (during free-fall) when the sensor begins to operate. Single mode fiber greatly reduces internal fiber interference between wave propagation modes so the source of the interference pattern observed is primarily due only to the changing index of refraction. ${ }^{7}$ A compact helium-neon $(\mathrm{HeNe})$ laser with a wavelength of $632.8 \mathrm{~nm}$ was selected 
as the light source, owing to the availability of both the laser and compatible fiber components at this wavelength. The laser provides a coherence length greater than $5 \mathrm{~cm}$ which is sufficient for simple interferometry.

Due to size constraints, the piston was designed to have a $4.25 \mathrm{~cm}$ diameter, allowing for a minimization of the actuation distance. Piston actuation was controlled using a $3.4 \mathrm{~N}-\mathrm{m}$ servo capable of delivering $\sim 760 \mathrm{~N}$ of linear force through a rack and pinion design. The piston had approximately $1.25 \mathrm{~cm}$ of actuation, resulting in approximately $18 \mathrm{~mL}$ of compression during a time interval of approximately $525 \mathrm{~ms}$. During the course of several static tests the piston demonstrated the capacity to endure a pressure up to $267 \mathrm{kPa}$, which is much larger than the maximum $67 \mathrm{kPa}$ encountered during dynamic actuation.

The gas cell (including all associated tubing) has an internal volume of approximately $60 \mathrm{~mL}$, and both tanks had a volume of approximately $50 \mathrm{~mL}$. During validation testing, one of the tanks was kept completely empty while the other was filled with $10-30 \mathrm{~mL}$ of water. For initial tests air was used as the sample (cover) gas. Air has a molar refractivity of $4.606 \times 10^{-6} \mathrm{~m}^{3} / \mathrm{mol}$ at $287.5 \mathrm{~K}$ (from Ref. [8]). The molar refractivity of air is relatively low compared to heavier gasses such as xenon, which has a molar refractivity of $11.04 \times 10^{-6} \mathrm{~m}^{3} / \mathrm{mol}$ computed based on a refractive index $\mathrm{n} \sim 1.0007$ (from Ref. [9]). While this does decrease the system sensitivity, the use of air as the sample gas greatly reduces the complexity of the device.

The HeNe laser (JDS Model 1007) is powered at 12 VDC by the primary payload battery. Single mode fiber splitters (Thorlabs, FC632-50B-FC) were used to construct the test apparatus. The hardware used for assembly of the optical system had limited angular adjustment capability for the fiber coupling hardware. Consequently, the fiber launch stages that coupled free-beam laser light into fiber optic cables were limited in their efficiency. Approximately $100 \mu \mathrm{W}$ of the $800 \mu \mathrm{W}$ laser $(12.5 \%)$ was successfully coupled into the fiber connecting the laser to the interferometer using a FiberPort (Thorlabs, PAF-X-11-PC-B) mounted directly to the laser output. Roughly 5\% of that light passed through the gas cell and was coupled into a second detection fiber using another FiberPort. The resulting final output of interferometer was about $4-6 \mu \mathrm{W}$. Despite all the losses, the photodiode detector (Thorlabs, PDA36A with built-in gain adjustment) used in the experiment was capable of measuring the interference pattern signal, yielding a high signal-to-noise ratio on the fringes.

\section{Results and Discussion}

\section{A. Proof of Concept Testing}

Before the final sounding rocket payload was fabricated, proof-of-concept testing was conducted using the setup shown in Fig. 4, where a HeNe laser (Thorlabs, HGR005, $543.4 \mathrm{~nm}$ ) and free-space optics were employed.

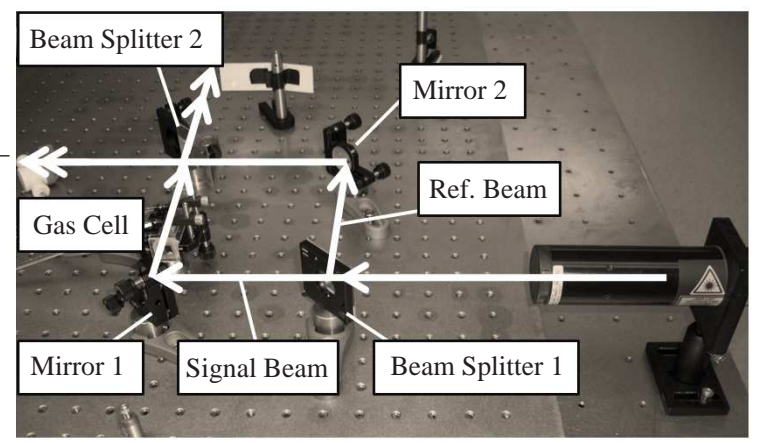

Figure 4. Proof-of-concept experiment using free-space optics.

Two distinct fringe shifts are required to calculate the volume of liquid contained within a single tank: a reference fringe shift and a fringe shift measured when a tank is open to the system. Like the final flight unit, the proof-ofconcept test setup consisted of two different tanks containing different volumes of liquid. Consequently, three sets of fringe shifts were measured: the reference, the fringe shift that occurs when Tank 1 is exposed to the system, and the fringe shift that occurs when Tank 2 is exposed to the system. The three sets of measurements obtained are displayed in Figure 5. The test parameters and results are recorded in Tables $1 \& 2$. The proof of concept tests were performed without equalizing the pressure after each piston cycle as required by Eq. 9 making an analysis by this equation invalid. For the sake of expediency, Boyle's law was instead used to approximate the unknown volumes.

As expected according to Eq. 2, the larger the ullage volume, the lower the fringe count. The isolated gas cell, 
Table 1. Parameters from the proof-of-concept testing.

\begin{tabular}{c|l}
\hline \hline$\lambda$ & $543.4 \mathrm{~nm}$ \\
\hline$A$ & $4.606 \times 10^{-6}\left(\mathrm{~m}^{3} / \mathrm{mol}\right)[8]$ \\
\hline$R$ & $8.32 \mathrm{~J} /(\mathrm{K} \cdot \mathrm{mol})$ \\
\hline$l$ & $0.05 \mathrm{~m}$ \\
\hline$T$ & $297 \mathrm{~K}$ \\
\hline$p$ & $101,325 \mathrm{~Pa}$ \\
\hline \hline
\end{tabular}

Table 2. Tabulated results from the proof-of-concept testing.

\begin{tabular}{c|l|l|l}
\hline \hline \multicolumn{1}{c|}{ Item } & Fringe Count & $\Delta \mathrm{p}(\mathrm{kPa})$ & Calculated Volume $(\mathrm{mL})$ \\
\hline \hline Reference (gas cell) & 15 & 56.4 & 34 \\
\hline Tank 1 (empty) & 5.75 & 22.4 & 51 \\
\hline Tank 2 (water) & 7 & 27.2 & 37 \\
\hline \hline
\end{tabular}
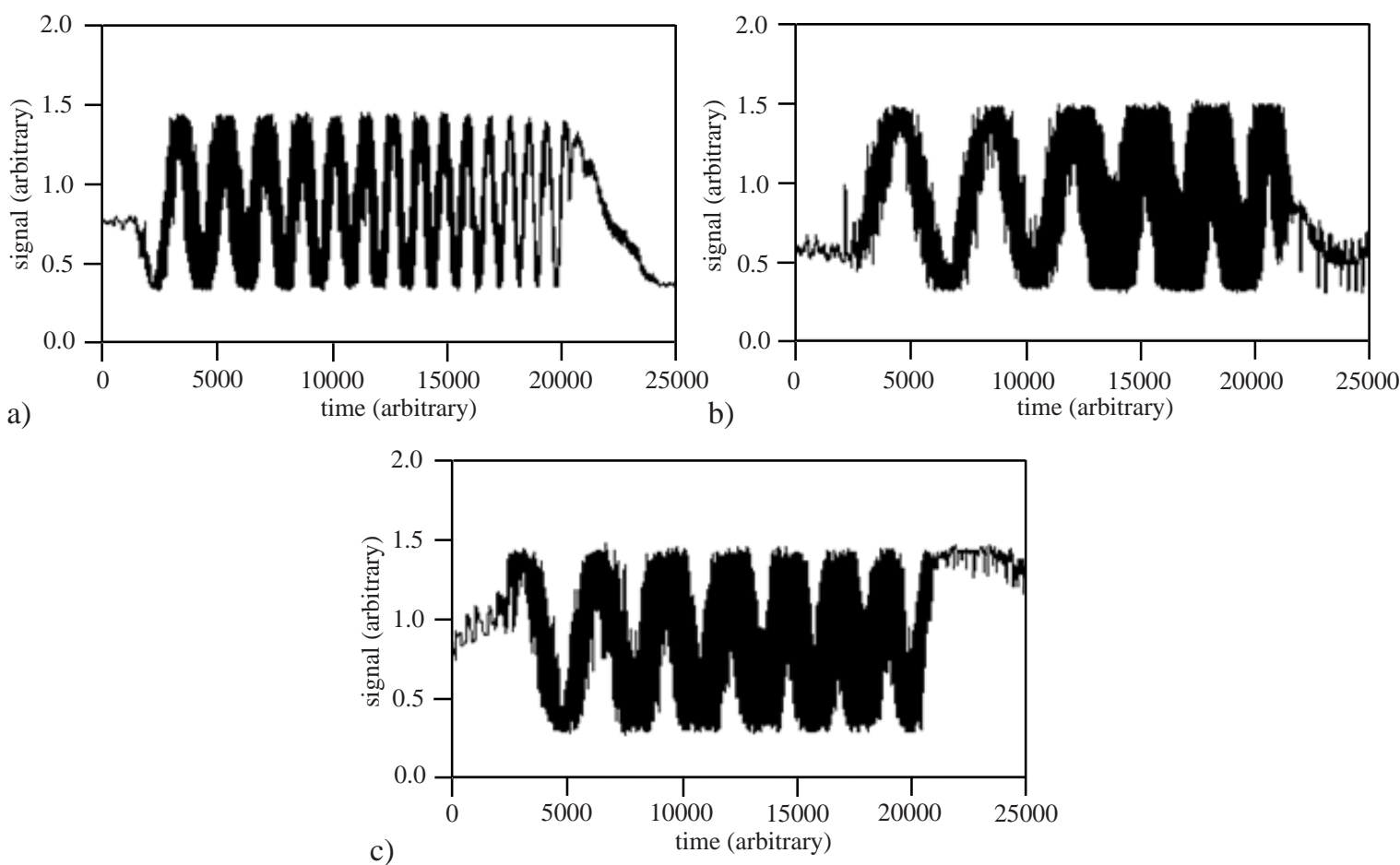

Figure 5. Proof-of-concept fringe-shift measurements for the a) reference case, b) case where Tank 1 (empty tank) was exposed to the apparatus, and c) case where Tank 2 (partially filled tank) was exposed to the apparatus.

having the smallest volume, has the greatest fringe count. The empty tank, having the largest ullage volume, yields the smallest number of fringes. The partially filled tank has an ullage volume in between the empty tank and the isolated gas cell, yielding fewer fringes than the former but more than the latter. The measurements indicate that the amount of liquid contained within this tank was $14 \mathrm{~mL}$. The actual amount of liquid contained within the tank was $11.5 \mathrm{~mL}$, resulting in an overall measurement error of roughly $20 \%$.

The large error observed during proof of concept testing is mostly a result of the fringe counts being obtained visually, without the aid of computer software. Additional contributions to the uncertainty include assumptions regarding the pressure and temperature of the gas and the estimation of the sensing path length. Although we used Boyle's law for analysis during the proof of concept tests, Eq. 9 is superior when determining unknown volumes as 
this method only requires the fringe count values (as determined by the interferometer) as opposed to requiring the stagnant pressure value and pressure changes (which are measured indirectly). The fully-assembled prototypical mass gauging sensor equalizes internal pressure before each piston compression by simultaneously cycling all solenoid valves allowing analysis by Eq. 9 .

\section{B. Laboratory Measurements Using Sounding Rocket Payload}

In this section we describe test results from the fiber optic system fabricated for flight on a sounding rocket (system in Fig. 3). Experimental data are presented in Tables 3 - 5 and Fig. 6.

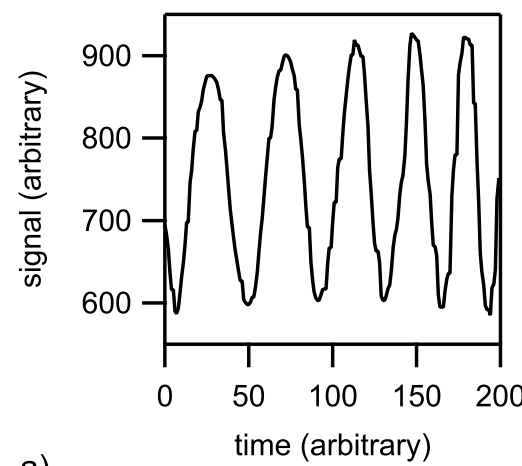

a)

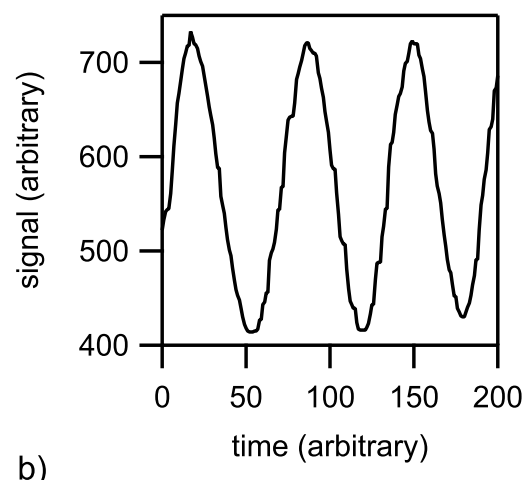

b)

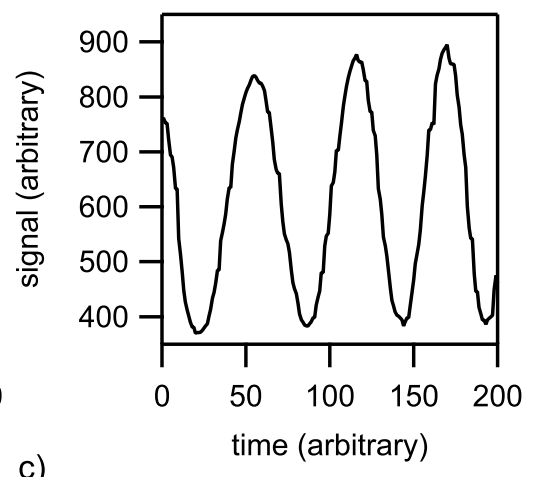

c)

Figure 6. Fringe shifts measured with the flight-test apparatus for a) the reference case, b) the case where the liquid tank was empty, and c) the case where the tank contained a level of liquid.

The volume of liquid in Tank 2 as measured by the sensor was $10.33 \mathrm{~mL}$. The actual volume of liquid was 10.53 $\mathrm{mL}$, giving an error on that particular measurement of less than $2 \%$. Obtaining an accurate count of the number of fringes was a more problematic issue in general, with accuracy on the fringe counts of \pm 0.25 fringes, implying an overall systematic error on the experimental setup of approximately $1.5 \mathrm{~mL}$. Difficulty in obtaining an accurate fringe count was largely due to significant noise in the interferometer output caused by the mechanical vibrations of the servo used to actuate the piston. Measuring the low-power output signal required an increase in the photodetector gain from $50-70 \mathrm{~dB}$, resulting in a poor signal-to-noise ratio. Recorded signals were filtered to remove high frequency noise and aid in fringe counting. The HeNe laser fluctuated in power during each piston cycle; these power fluctuations were most likely caused by back reflection in the fiber-optics which could be alleviated in the future by installing a fiber-optic isolator. Even by visual inspection, it was readily seen that there were some inconsistencies in the fringe counts between each piston cycle. These inconsistencies were most likely due to a pressure leak somewhere in the system, making it difficult to reproduce results with each piston cycle.

Table 3. Parameters for the testing of the payload apparatus.

\begin{tabular}{c|l}
\hline \hline$\lambda$ & $632.8 \mathrm{~nm}$ \\
\hline$A$ & $4.606 \times 10^{-6}\left(\mathrm{~m}^{3} / \mathrm{mol}\right)[9]$ \\
\hline$R$ & $8.32 \mathrm{~J} /(\mathrm{K} \cdot \mathrm{mol})$ \\
\hline$l$ & $0.0432 \mathrm{~m}$ \\
\hline$T$ & $297 \mathrm{~K}$ \\
\hline$p$ & $101,900 \mathrm{~Pa}$ \\
\hline \hline
\end{tabular}

Obtaining accurate results was also made problematic due to the fact that the volumes measured by our optical mass gauge were very small, and a small ullage volume difference of $10 \mathrm{~mL}$ corresponded to a fringe shift difference of approximately 0.3 fringes. In the present test apparatus, the relatively weak dependence of fringe count on the tank volume limits the achievable sensitivity. In the future, using a sample gas with a higher index of refraction (therefore yielding higher fringe counts) could help alleviate this issue. 
Table 4. Volume of liquid in Tank 2 from testing of the payload apparatus.

\begin{tabular}{l|l}
\hline \hline Measured Liquid Volume (mL) & 10.33 \\
\hline Actual Liquid Volume (mL) & 10.53 \\
\hline Percent Error & $1.90 \%$ \\
\hline \hline
\end{tabular}

Table 5. Number of fringe shifts measured for each test case from testing of the payload apparatus.

\begin{tabular}{l|l|l|l}
\hline \hline & Reference & Tank 1 (empty) & Tank 2 (water) \\
\hline \# of Fringes & 5.33 & 3.30 & 3.59 \\
\hline \hline
\end{tabular}

\section{Vibration Test}

A full scale vibration test of the optical mass gauge payload was performed on a vibration table (Sierra Nevada Corporation, Louisville, CO). The payload was exposed to several different tests: two tests in the Z-Axis (sine sweep and random), and a random test in the $\mathrm{X}$ and $\mathrm{Y}$ axes. None of the components showed signs of damage upon visual inspection, and the optical components remained in alignment (although there was a slight laser coupling efficiency loss). In each test, the vibration table was ramped up to a maximum of $7 \mathrm{G}$ 's for no more than 30 seconds. The payload's first mode of resonant frequency was at approximately $200 \mathrm{~Hz}$, and briefly experienced 25G's at this frequency.

\section{Future Work}

There is much room for additional improvements in future iterations of the optical mass gauge sensor. For example, higher-quality optical components such as NBK-7 windows (or similar) could be used in place of the lower-quality commercial window glass presently used for the gas cell windows. Also, selecting an optical splitter that yields a different split ratio (50:50 in the current design) would serve to provide a stronger signal beam, resulting in greater fringe visibility. Finally, replacing the current fiber coupling hardware used on the gas cell with more permanently mounted fiber couplers would aid in alignment stability of the optics, especially as the payload experiences shock during launch.

Methods to isolate the mechanical vibrations from the optical components are being investigated. The current iteration of the device suffers from the mechanical vibrations of the servo used to actuate the piston, producing significant noise in the interferometer output.

In the present work, the unfiltered signal was acquired using an analog input on the microcontroller operating in a DC-coupled mode. Filtering the signal or operating in an AC-coupled mode may have allowed for removal of noise from the measurement, and is simple enough to implement on future iterations of this work. Another noise rejection technique that may be employed is to pulse or chop a laser beam above the noise threshold of the system, and then measure the signal using an AC-coupled scope or lock-in amplifier. This would allow filtering below the frequency threshold of the noise while passing the signal through to the data acquisition system, which could be very useful on a launch vehicle where vibrational noise can prove to be an impediment to performing optical interferometric measurements.

While this experiment was constrained in size and mass, a more accurate system producing a larger fringe count could, in principle, be fabricated if these constraints were less severe. A general difficulty of this system is the need to keep the liquid from entering the gas cell. One potential solution that was not tested is to install a metal 'mesh' that allows gas to enter the gas cell while preventing liquid from entering through its surface tension.

\section{Conclusion}

The fiber optic-coupled interferometric mass gauging system presented in this paper has many advantages over other proposed or in-use on-orbit mass gauging techniques. The sensing technique is compact, rugged, and requires only a small displacement volume with minimal modification to existing tankage. Such a sensor can theoretically operate in any gravitational environment, including microgravity, eliminating the need for spacecraft acceleration (settling) prior to a liquid volume measurement. Data obtained using a prototypical fiber-coupled optical mass gauge for flight testing on a sounding rocket platform consistently correlated with the various liquid volumes contained in 
a multi-tank system. While the overall random uncertainty for the current system is $\pm 1.5 \mathrm{~mL}$, in one laboratory test of the sensor a liquid level of $10.53 \mathrm{~mL}( \pm 2 \%)$ was measured. In general, the system shows good quantitative and excellent qualitative correlation between ullage volume and fringe count.

\section{Acknowledgments}

The authors of this paper would like to thank Omnis, Inc. for their generous financial donation that seeded our research efforts. Financial support was also received from the Colorado State University Senior Design Program, the Colorado State University NASA Space Grant Consortium, and Masten Space Systems, Inc. We would like to thank Jason Priebe, Dan Goodrich, and Lad Kurtis of Sierra Nevada Corp. for the use of their vibration table. We acknowledge technical guidance from Prof. John Williams, Dr. Sachin Joschi, and graduate students Lei Tao, Brian Lee and Frank Loccisano.

\section{References}

\footnotetext{
${ }^{1}$ Jurns, J.M., and Rogers, A.C., “Compression Mass Gauge Testing in a Liquid Hydrogen Dewar," NASA Contractor Report CR-198366, Lewis Research Center, Cleveland, OH, July 1995.

${ }^{2}$ Haberbusch, M., "Flexible Cryogenic Temperature and Liquid-Level Probes," NASA Tech Brief SSC-00191, Stennis Space Center, Nov. 2005.

${ }^{3}$ Nurge, M., and Youngquist, R., “Capacitive Sensors for Measuring Masses of Cryogenic Fluids,” NASA Tech Brief KSC-12457, Kennedy Space Center, Cape Canaveral, FL, Sept. 2003.

${ }^{4}$ Dodge, F., Green, S., Petullo, S., et al., "Development and Design of a Zero-g Liquid Quantity Gauge for Solar Thermal Vehicle," NASA/TP_ 2002-211595, Glenn Research Center, Cleveland, OH, Nov. 2002.

${ }^{5}$ Justak, J., Caimi, F.M., Bryant, C.B., et al., "An Optical Mass Gauge Sensor for Zero-G Environments,” Advances in Cryogenic Engineering, CEC-2004, AIP Conf. Proc., Vol. 710, pp 451-460, 2004.

${ }^{6}$ Witherow, W., and Pedersen, K., "Mass Gauging Demonstrator for Any Gravitational Conditions," NASA Internal Research and Development, Marshall Space Flight Center, Huntsville, AL, Mar. 2007.

${ }^{7}$ Sinko, J.E., Korman, V., Hendrickson, A., et al., "A Miniaturized Optical Sensor for Leak Detection in a Space Environment," Journal of Spacecraft and Rockets, Accepted for Publication, Apr. 2010.

${ }^{8}$ Korman, V., Gregory, D.A., Banish, R.M., "Phase Transition Thermal Expansion Measurement Technique using a Modified Michelson Interferometer," J. Opt. A: Pure Appl. Opt., Vol. 6, pp 781-786, 2004.

${ }^{9}$ Born, M., Wolf, E., Principles of Optics, 7th expanded edition, Cambridge University Press, Cambridge, 1999.

${ }^{10}$ Arfaoui, I., Duguay, M.A., Lavoie, C., et al., "Tapered Hollow Optical Fibers with a Xenon Core for WDM Applications" Applications of Photonic Technology, Proc. SPIE, Vol. 4833, pp 1021-1029, 2003.
} 